Pacific Journal of Mathematic 


\title{
AUTOMORPHISMS OF DIMENSION GROUPS AND THE CONSTRUCTION OF AF ALGEBRAS
}

\section{Chao-Liang SHEN}

\begin{abstract}
Recent results of Edward G. Effros and the author show that if a dimension group is simple, totally ordered and with underlying group $Z^{n}$, then we can construct explicitly an $\mathrm{AF} C^{*}$-algebra with the given group as its $K_{0}$ by using the Jacobi-Perron algorithm. While the Jacobi-Perron algorithm breaks down for nontotally ordered groups, we study the construction problem via the consideration of automorphisms of the dimension group. We find the necessary and sufficient condition for a nontotally ordered simple dimension group $\left(Z^{3}, P_{(1, \alpha, \beta)}\right)$ being stationary is that both $\alpha$ and $\beta$ lie in the same quadratic number field. We also provide an explicit method for constructing Bratteli diagrams (and hence corresponding $A F \quad C^{*}$-algebras) for this type of groups.
\end{abstract}

Introduction. Since George Elliott introduced dimension theory for approximately finite $C^{*}$-algebras, considerable progress has been made in the study of AF $C^{*}$-algebras ([3], [4], [5], [6], [7], [8], [2]). In [5] and [6], Effros and the author raised the question of constructing AF algebras with given dimension groups as their $K_{0}$, and answered it in the case when the given dimension group is simple totally ordered and with underlying group $Z^{n}$ by using the Jacobi-Perron algorithm. Based on this and some examples of nontotally ordered simple dimension groups $([6, \S 4])$. we conjectured for any simple dimension group $G$ with underlying group $Z^{n}$, both that there exists an inductive sequence

$$
\boldsymbol{Z}^{n} \stackrel{\varphi_{1}}{\longrightarrow} \boldsymbol{Z}^{n} \stackrel{\varphi_{2}}{\longrightarrow} \boldsymbol{Z}^{n} \longrightarrow \cdots
$$

where $\varphi_{k} \in \mathrm{GL}(n, \boldsymbol{Z})$ with nonnegative entries such that $\lim _{\longrightarrow}\left(\boldsymbol{Z}^{n}, \varphi_{k}\right) \cong G$ and that there exists effective methods for constructing these $\varphi_{k}$ 's. In the meantime, our results have been applied by Cuntz, Krieger, Pimsner and Voiculescu ([2], [11]) to problems in topological Markov chains and to the irrational rotation $C^{*}$-algebras.

While the recent work of Riedel [12] supports the first part of conjecture, the construction problem still remains. Some dimension groups which are certainly worth first consideration are those having unique state (see [4] for definition). In this direction, as motivated by the work of Cuntz and Krieger, we ask the following question about existence and construction: 
Question EC: "Find a necessary and sufficient condition for a given simple dimension group $\left(\boldsymbol{Z}^{n}, P\right)$ to be the inductive limit of a sequence (which shall be called a stationary Elliott system)

$$
\boldsymbol{Z}^{n} \stackrel{\varphi}{\longrightarrow} \boldsymbol{Z}^{n} \stackrel{\varphi}{\longrightarrow} \cdots
$$

with constant connecting mapping $\varphi \in \mathrm{GL}(n, \boldsymbol{Z})$ of nonnegative entries, and find a method for constructing the "Bratteli diagram" $\varphi$."

Dimension groups arising in this fashion are called stationary dimension groups. All stationary dimension groups have exactly one state [6, Prop. 2.2].

In this paper we answer Question EC for the case $n=3$ and when $\left(Z^{3}, P\right)$ is not totally ordered ( $n=2$ case is completely answered by [5]). In our study we find that the answer to this question is closely related to the automorphism group of the given dimension group. In fact, we show that the structure of AUT $\left(Z^{3}, P\right)$ contains rich information, and the "Bratteli diagram" $\varphi$ "hides" inside $\operatorname{AUT}\left(Z^{3}, P\right)$ (see $\S 2$ for detail). While the Jacobi-Perron algorithm breaks down for nontotally ordered groups (see [6, §4]), and no periodicity condition for multidimensional continued fraction is known, if our approach can be generalized to $n \geqq 4$ case, it will be of great interest for the construction theory of AF algebras. Such a generalization might even solve the periodicity problem of simultaneous Diophantine approximation posed by Lagrange (see $[10$, $\S 10.12])$. In $\S 2$ we state our philosophy for attacking the problem and prove our main theorems. Finally we mention some open problems motivated by our method. We refer readers to the paper [3], [4], [5], [6], [7], [8], [13] and [14] for relevant results and definitions.

1. Some preliminaries. Let $G$ be a dimension group. An automorphism of $G$ is a group automorphism $\psi$ such that $\psi, \psi^{-1}$ are positive homomorphisms. $G$ is simple if it has no nontrivial order ideal. Given a simple dimension group $G$ with underlying group $\boldsymbol{Z}^{2}$, then $G$ is isomorphic to the dimension: group $\left(\boldsymbol{Z}^{2}, P_{\alpha}\right)$, where $\alpha \in \boldsymbol{R}_{+} \mid \boldsymbol{Q}, \quad P_{\alpha}=\left\{(x, y) \in \boldsymbol{Z}^{2}: \alpha x+y \geqq 0\right\}$ is the positive cone. Two such groups $\left(Z^{2}, P_{\alpha}\right),\left(Z^{2}, P_{\beta}\right)$ are isomorphic if and only if the continued fraction expansion of $\alpha$ and $\beta$ agree after finite stage ([5]).

Let $\alpha \in \boldsymbol{R}_{+} \mid \boldsymbol{Q}$, be a quadratic irrational, and let

$$
\left[a_{0}, a_{1}, \cdots, a_{n}, \cdots\right]
$$

be its simple continued fraction expansion [10, Ch. X]. Then it is periodic. Replacing by equivalent irrational if necessary, we may assume $\alpha>1$, and the period of the expansion is $\left[a_{0}, \cdots, a_{L-1}\right]$. We 
have proved the following

Theorem 1.1 ([13, Theorems 2.1, 2.3]). Given $\alpha \in \boldsymbol{R}_{+} \mid \boldsymbol{Q}$. Then the automorphism group AUT $\left(Z^{2}, P_{\alpha}\right)$ is nontrivial if and only if $\alpha$ is quadratic. Furthermore, if $\alpha$ is quadratic (and assume its period starts from $\left.a_{0}\right)$, then $\mathrm{AUT}\left(\boldsymbol{Z}^{2}, P_{\alpha}\right)$ is cyclic and is generated by the canonical automorphism

$$
\psi_{\alpha}=\left[\begin{array}{ll}
p_{L-1} & q_{L-1} \\
p_{L-2} & q_{L-2}
\end{array}\right],
$$

where $p_{k}, q_{j}$ are defined inductively by

$$
\begin{aligned}
& p_{0}=a_{0}, \quad p_{1}=a_{1} a_{0}+1, \cdots, p_{n}=a_{n} p_{n-1}+p_{n-2} \\
& q_{0}=1, \quad q_{1}=a_{1}, \cdots, q_{n}=a_{n} q_{n-1}+q_{n-2} .
\end{aligned}
$$

If $k>0$,

$$
\psi_{\alpha}^{k}=\left[\begin{array}{ll}
p_{k L-1} & q_{k L-1} \\
p_{k L-2} & q_{k L-2}
\end{array}\right]
$$

Given a simple dimension group $G$ with underlying group $Z^{n}$. Then $G=H \bigoplus_{t} Z^{p}$, where $p \geqq 0$, where $H$ is a dense subgroup of $\boldsymbol{R}^{d}$ for some $d \geqq 1$ (see [4, $\left.\S 4\right]$, [6]), and the ordering is given by $(h, z) \geqq 0$ if $h>0$, or $h=z=0$. In particular, if $G$ has underlying group $\boldsymbol{Z}^{3}$, with unique state and is not totally ordered, then there exists an $\alpha \in \boldsymbol{R}_{+} \mid \boldsymbol{Q}$ such that $G \cong\left(\boldsymbol{Z}^{2}, P_{\alpha}\right) \bigoplus_{t} \boldsymbol{Z}$.

The following proposition will be useful

Proposition 1.2. Automorphisms of $G=\left(Z^{2}, P_{\alpha}\right) \bigoplus_{t} Z$ are of the form

$$
\psi=\left[\begin{array}{lll}
a & b & 0 \\
c & d & 0 \\
e & f & g
\end{array}\right]
$$

where $\psi \in \operatorname{GL}(3, \boldsymbol{Z})$, and $\left[\begin{array}{ll}a & b \\ c & d\end{array}\right] \in \operatorname{AUT}\left(\boldsymbol{Z}^{2}, P_{\alpha}\right)$.

Proof. If

$$
\psi=\left[\begin{array}{lll}
a_{11} & a_{12} & a_{13} \\
a_{21} & a_{22} & a_{23} \\
a_{31} & a_{32} & a_{33}
\end{array}\right] \in \operatorname{AUT}(G)
$$

then 


$$
\begin{aligned}
(x, y, z) & \in G_{+} \backslash\{0\} \\
& \Longleftrightarrow \alpha\left(a_{11} x+a_{12} y+a_{13} z\right)+\left(a_{21} x+a_{22} y+a_{23} z\right)>0 \\
& \Longrightarrow\left(a_{11} \alpha+a_{21}\right) x+\left(a_{12} \alpha+a_{22}\right) y+\left(a_{13} \alpha+a_{23}\right) z>0 .
\end{aligned}
$$

Thus we must have

$$
\begin{gathered}
a_{12} \alpha+a_{22}>0, \\
\frac{\left(a_{11} \alpha+a_{21}\right)}{\left(a_{12} \alpha+a_{22}\right)}=\alpha, \\
a_{13} \alpha+a_{23}=0 .
\end{gathered}
$$

Since $\alpha$ is irrational, (1.3) implies $a_{13}=a_{23}=0$. Thus $a_{33}= \pm 1$, and $\left[\begin{array}{ll}a_{11} & a_{12} \\ a_{21} & a_{22}\end{array}\right] \in \operatorname{AUT}\left(Z^{2}, P_{\alpha}\right)([14$, Lemma 4.7]). Conversely if $\psi$ is of the form (1.0) with $\left[\begin{array}{ll}a & b \\ c & d\end{array}\right] \in \operatorname{AUT}\left(\boldsymbol{Z}^{2}, P_{\alpha}\right)$, direct computation shows that is $\operatorname{AUT}(G)$.

The following lemma is straight forward, and will be useful for our discussion.

Lemma 1.3. If $G, H$ are dimension groups, and $\Phi: G \rightarrow H$ is an isomorphism (in order sense), then $\operatorname{AUT}(H)=\Phi \operatorname{AUT}(G) \Phi^{-1}$.

Thus if $\operatorname{AUT}(G)$ is nontrivial, then so is $\operatorname{AUT}(H)$, and conversely. Suppose we are given a stationary Elliott system

$$
\boldsymbol{Z}^{n} \stackrel{\varphi}{\longrightarrow} \boldsymbol{Z}^{n} \stackrel{\varphi}{\longrightarrow} \cdots
$$

where $\varphi$ is a strictly positive matrix in GL $(n, \boldsymbol{Z})$. (From now on we shall use the notation $\mathrm{GL}(n, \boldsymbol{Z})_{+}$to denote the set of unimodular. matrices with nonnegative entries, $\mathrm{GL}(n, Z)_{++}$to denote the subset of $\varphi$ in $\mathrm{GL}(n, \boldsymbol{Z})_{+}$such that $\varphi^{m}$ is strictly positive for sufficiently large $m$.) Then the resulting stationary dimension group has unique state, and we can express its positive cone as

$$
\begin{aligned}
P_{\left(1, \alpha_{2}, \cdots, \alpha_{n}\right)}= & \left\{\left(x_{1}, \cdots, x_{n}\right) \in \boldsymbol{Z}^{n}: x_{1}+\alpha_{2} x_{2}+\cdots+\alpha_{n} x_{n}>0\right\} \\
& \cup\{(0, \cdots, 0)\},
\end{aligned}
$$

where $\left(1, \alpha_{2}, \cdots, \alpha_{n}\right)$ is the eigenvector of the Perron eigenvalue of $\varphi^{t r}$, at least one of $\alpha_{i}$ is irrational, and $\alpha_{2}, \cdots, \alpha_{n}>0$. In fact the same result is true if $\varphi \in \operatorname{GL}(n, \boldsymbol{Z})_{++}$. Since for $\varphi$ nonnegative, $\varphi$ has an eigenvalue $\lambda$ with $\lambda \geqq|\alpha|$ for all other eigenvalues $\alpha$ (see [9], Ch. III, §3). On the other hand, $\varphi^{m}$ is strictly positive, it has an eigenvalue $\mu$ with $\mu>|\beta|$ for all other eigenvalues $\beta$. Using the fact that $\operatorname{sp}\left(\varphi^{t r}\right)^{m}=\left(\operatorname{sp} \varphi^{t r}\right)^{m}$, we conclude that $\mu=\lambda^{m}$; again 
$\left(1, \alpha_{2}, \cdots, \alpha_{n}\right)$ is the eigenvector of $\varphi^{m}$ corresponding to the Perron eigenvalue, and hence $\alpha_{i}>0$ for all $i$. Since the Perron eigenspace is one dimensional, $\left(1, \alpha_{2}, \cdots, \alpha_{n}\right)$ must be the unique Perron vector of $\varphi$ of that form.

Although the following lemma is not difficult, we give a proof here for completeness. $\varphi \in \mathrm{GL}(3, \boldsymbol{Z})_{+}$.

Lemma 1.4. If the stationary dimension group $G=\lim \left(\boldsymbol{Z}^{3}, \varphi\right)$ is simple, and is not totally ordered, then the Perron eigenvalue $\lambda$ of $\varphi$ is a quadratic integral unit, i.e., it satisfies an equation of the form $x^{2}-b x+c=0$, where $b \in Z, c= \pm 1$.

Proof. It is easy to see (c.f. [1, Cor. 3.5]) that $G$ is simple if and only if $\varphi \in \mathrm{GL}(3, \boldsymbol{Z})_{++}$. Write $G=\left(\boldsymbol{Z}^{3}, P_{\left(1, \alpha_{1}, \alpha_{2}\right)}\right)$, where $\left(1, \alpha_{1}, \alpha_{2}\right)$ is the Perron eigenvector of $\phi$. Then by computation we see that $\alpha_{1}=P(\lambda) / R(\lambda), \alpha_{2}=Q(\lambda) / R(\lambda)$, where $R(x)$ is a linear polynomial, and one of $P(x)$ and $Q(x)$ is linear, and the other is quadratic. Since $G$ is not totally ordered, $1, \alpha_{1}$ and $\alpha_{2}$ are rationally dependent. This implies that the monic minimal polynomial $m(x)$ of $\lambda$ over $\boldsymbol{Q}$ is quadratic, it divides the characteristic polynomial $X(x)$ of $\varphi$, and $X(x) \in Z[x]$ is monic. Write $X(x)=m(x) q(x), q(x) \in \boldsymbol{Q}[x]$. Since $\boldsymbol{Z}$ is a normal integral domain, $m(x) \in Z[x]$. On the other hand, since the constant term of $X(x)$ is \pm 1 , the constant term of $m(x)$ must be 1 or -1 . Thus we conclude that $\lambda$ is a quadratic integral unit. Further calculation shows that we can write $\alpha_{i}$ as $r_{i}+s_{i} \lambda$, where $r_{i}, s_{i} \in \boldsymbol{Q}$.

2. The main theorems. We shall first state and prove one part of Theorem 2.1. Then we explain our idea and prove a technical lemma. The proof of the theorem and the construction of the Bratteli diagram will be continued after Lemma 2.2 .

THeOREM 2.1. The simple dimension group $\left(\boldsymbol{Z}^{2}, P_{\alpha}\right) \bigoplus_{t} \boldsymbol{Z}$ is isomorphic to a stationary dimension group if and only if $\alpha$ is a quadratic irrational.

Proof. Here we prove the "only if" part. Suppose $\left(\boldsymbol{Z}^{2}, P_{\alpha}\right) \bigoplus_{t} \boldsymbol{Z}$ is isomorphic to a stationary dimension group $G=\lim \left(\boldsymbol{Z}^{3}, \varphi\right), \varphi \in$ $\mathrm{GL}(3, \boldsymbol{Z})_{++}$. Write

$$
\left.G_{+}=\left\{(x, y, z) \in Z^{3}: x+\alpha_{2} y+\alpha_{2} z>0\right\} \cup\{0,0,0)\right\},
$$

where $\left(1, \alpha_{2}, \alpha_{3}\right)$ is the Perron eigenvector of $\phi^{t_{r}}$. Then $1, \alpha_{2}, \alpha_{3}$ are rationally dependent since $\left(\boldsymbol{Z}^{2}, P_{\alpha}\right) \bigoplus_{t} \boldsymbol{Z}$ is not totally ordered. 
By Lemma 1.4, we can write $\alpha_{i}=r_{i}+s_{i} \lambda$, where $r_{i}, s_{i} \in \boldsymbol{Q}$, and $\lambda$ is the Perron eigenvalue of $\varphi^{t r}$, which is a quadratic integral unit. Let

$$
\Phi=\left[a_{\imath j}\right]_{i, j=1}^{3}:\left(\boldsymbol{Z}^{2}, P_{\alpha}\right) \bigoplus_{t} Z \longrightarrow G
$$

be an isomorphism. Then $(x, y, z) \in\left[\left(\boldsymbol{Z}^{2}, P_{\alpha}\right) \bigoplus_{t} \boldsymbol{Z}\right]_{+} \backslash\{(0,0,0)\}$ if and only if $\left(a_{11} x+a_{12} y+a_{13} z, a_{21} x+a_{22} y+a_{23} z, a_{31} x+a_{32} y+a_{33} z\right) \in G_{+} \backslash\{(0,0,0)\}$, i.e., $\left(a_{11}+a_{21} \alpha_{2}+a_{31} \alpha_{3}\right) x+\left(a_{12}+a_{22} \alpha_{2}+a_{32} \alpha_{3}\right) y+\left(a_{13}+a_{23} \alpha_{2}+a_{33} \alpha_{3}\right) z>0$. Since $(x, y, z) \in\left[\left(\boldsymbol{Z}^{2}, P_{\alpha}\right) \bigoplus_{t} \boldsymbol{Z}\right]_{+} \backslash\{(0,0,0)\}$, we have $\alpha x+y>0$. Hence $a_{13}+a_{23} \alpha_{2}+a_{33} \alpha_{3}=0, a_{12}+a_{22} \alpha_{2}+a_{32} \alpha_{3}>0$ and $\left(a_{11}+a_{21} \alpha_{2}+a_{31} \alpha_{3}\right) /$ $\left(a_{12}+a_{22} \alpha_{2}+a_{32} \alpha_{3}\right)=\alpha . \quad \alpha_{2}, \alpha_{3} \in \boldsymbol{Q}(\lambda)$ imply that $\alpha \in \boldsymbol{Q}(\lambda)$. The fact that $\lambda$ being quadratic implies that $\alpha$ is a quadratic irrational. This proves the "only if" part of the theorem.

Now suppose we are given the simple dimension group $\left(\boldsymbol{Z}^{2}, P_{\alpha}\right) \bigoplus_{t} \boldsymbol{Z}$, where $\alpha$ is quadratic. Our idea for proving that $\left(\boldsymbol{Z}^{2}, P_{\alpha}\right) \bigoplus_{t} \boldsymbol{Z}$ isomorphic to a stationary dimension group is first to replace $\left(\boldsymbol{Z}^{2}, P_{\alpha}\right) \bigoplus_{t} \boldsymbol{Z}$ by a suitable isomorphic dimension group $G=\left(\boldsymbol{Z}^{3}, P_{\left(1, \alpha_{2}, \alpha_{3}\right)}\right)$, where $\alpha_{2}, \alpha_{3} \in \boldsymbol{R}_{+} \backslash \boldsymbol{Q}$, and then to prove that the automorphism group AUT $(G)$ of $G$ is nontrivial. Furthermore we prove that AUT $(G)$ contains an element $\varphi \in \mathrm{GL}(3, \boldsymbol{Z})_{++}$, and for this $\varphi,\left(1, \alpha_{2}, \alpha_{3}\right)$ is the Perron eigenvector of $\phi^{t r}$. For our purpose, we need the following

Lemma 2.2. The dimension group $H=\left(Z^{2}, P_{\alpha}\right) \bigoplus_{t} Z$ is isomorphic to dimension groups of the form

$$
\left(\boldsymbol{Z}^{3}, P_{\left(1, n_{1} \alpha+m, n_{2} \alpha\right)}\right),
$$

where $n_{1}, n_{2}, m \in Z$, g.c.d. $\left(n_{1}, n_{2}\right)=1$.

Proof. Choose $u, v \in Z$ such that $n_{1} u+n_{2} v=1$. Define

$$
\psi=\left[\begin{array}{ccc}
-m u & 1 & m n_{2} \\
u & 0 & -n_{2} \\
v & 0 & n_{1}
\end{array}\right] .
$$

Then $\psi \in \operatorname{GL}(3, Z)$ (in fact $\operatorname{det} \psi=-1$ ), and

$$
\psi^{-1}=\left[\begin{array}{ccc}
0 & n_{1} & n_{2} \\
1 & m & 0 \\
0 & -v & u
\end{array}\right]
$$

Now notice that if $(x, y, z) \in H_{+} \backslash\{(0,0,0)\}$, then $\psi(x, y, z) \in P \backslash\{(0,0,0)\}$. This because $\left(-m u x+y+m n_{2} z\right)+\left(n_{1} \alpha+m\right)\left(u x-n_{2} z\right)+n_{2} \alpha\left(v x-n_{1} z\right)=$ 
$\alpha x+y>0$. On the other hand, if $\left(x^{\prime}, y^{\prime}, z^{\prime}\right) \in P \backslash\{(0,0,0)\}$, then $\psi^{-1}\left(x^{\prime}, y^{\prime}, z^{\prime}\right) \in H_{+} \backslash\{(0,0,0)\}$. This is because $\psi^{-1}\left(x^{\prime}, y^{\prime}, z^{\prime}\right)=\left(n_{1} y^{\prime}+n_{2} z^{\prime}\right.$, $\left.x^{\prime}+m y^{\prime},-v y^{\prime}+u z^{\prime}\right)$, and $\alpha\left(n_{1} y^{\prime}+n_{2} z^{\prime}\right)+\left(x^{\prime}+m y^{\prime}\right)=x^{\prime}+\left(n_{1} \alpha+\right.$ $m) y^{\prime}+n_{2} \alpha z^{\prime}>0$. Thus $\psi: H \rightarrow\left(\boldsymbol{Z}^{3}, P_{\left(1, n_{1} \alpha+m, n_{2} \alpha\right)}\right)$ is an isomorphism.

As the "suitable isomorphic group", we will pick $G=\left(Z^{3}, P_{(1, \alpha, \alpha)}\right)$, namely we take $n_{1}=1, n_{2}=1, m=0$. Furthermore, we choose $u=1, v=0$, i.e., we choose

$$
\psi=\left[\begin{array}{rrr}
0 & 1 & 0 \\
1 & 0 & -1 \\
0 & 0 & 1
\end{array}\right]
$$

Now set $\left[a_{0}, a_{1}, \cdots\right]$ to be the continued fraction expansion of the quadratic irrational $\alpha$, and assume $\left[a_{0}, \cdots, a_{L-1}\right]$ is its period. By Theorem 1.1 and Proposition 1.2

$$
\Phi=\left[\begin{array}{lll}
p_{k L-1} & q_{k L-1} & 0 \\
p_{k L-2} & q_{k L-2} & 0 \\
\alpha & b & 1
\end{array}\right] \in \operatorname{AUT}\left(\left(\boldsymbol{Z}^{2}, P_{\alpha}\right) \bigoplus_{t} \boldsymbol{Z}\right),
$$

where $a, b \in \boldsymbol{Z}$ are arbitrary. By Lemma $1.3, \psi \Phi \psi^{-1} \in \operatorname{AUT}\left(\boldsymbol{Z}^{3}, P_{(1, \alpha, \alpha)}\right)$,

$$
\begin{aligned}
\Phi_{(k ; a, b)} & \equiv \psi \Phi_{\psi^{-1}}=\left[\begin{array}{rrr}
0 & 1 & 0 \\
1 & 0 & -1 \\
0 & 0 & 1
\end{array}\right]\left[\begin{array}{lll}
p_{k L-1} & q_{k L-1} & 0 \\
p_{k L-2} & q_{k L-2} & 0 \\
a & b & 1
\end{array}\right]\left[\begin{array}{lll}
0 & 1 & 1 \\
1 & 0 & 0 \\
0 & 0 & 1
\end{array}\right] \\
& =\left[\begin{array}{lll}
q_{k L-2} & p_{k L-2} & p_{k L-2} \\
q_{k L-1}-b & p_{k L-1}-a & p_{k L-1}-(a+1) \\
b & a & a+1
\end{array}\right] .
\end{aligned}
$$

Now we can continue the proof of Theorem 2.1.

Proof of Theorem 2.1 (continued): If we can choose suitable $k, a, b$ such that $\Phi_{(k ; a, b)} \in \mathrm{GL}(3, Z)_{++}$and $(1, \alpha, \alpha)$ is the Perron eigenvector of $\Phi_{(k ; a, b)}{ }^{t r}$, then we are done. For this purpose, we first fix an $a>0, a b>0$, and then with respect to these $a$ and $b$, we choose and fix a sufficiently large even number $k$ (the reason for choosing $k$ even is to make the $\operatorname{det} \Phi=p_{k L-1} q_{k L-2}-p_{k L-2} q_{k L-1}=(-1)^{k L}$ equal 1), such that $p_{k L-1}>a+1, q_{k L-1}>b$. This choice is possible since the sequences $\left\{p_{n}\right\},\left\{q_{n}\right\}$ are increasing to infinity. Then $\Phi_{(k ; a, b)} \in \mathrm{GL}(3, \boldsymbol{Z})_{++}$. On the other hand, since $\Phi_{(k ; a, b)} \in \operatorname{AUT}\left(\boldsymbol{Z}^{3}, P_{(1, \alpha, \alpha)}\right)$, $\left.(x, y, z) \in P_{(1, \alpha, \alpha)}\right)\{(0,0,0)\}$ if and only if $\left.\Phi_{(k ; a, b)}(x, y, z) \in P_{(1, \alpha, \alpha)}\right)\{(0,0,0)\}$, i.e., $\left(q_{k L-2} x+p_{k L-2} y+p_{k L-2} z\right)+\alpha\left[\left(q_{k L-1}-b\right) x+\left(p_{k L-1}-a\right) y+\left(p_{k L-1}-\right.\right.$ $(a+1)) z]+\alpha[b x+a y+(a+1) z]=\left(q_{k L-2}+\alpha q_{k L-1}\right) x+\left(p_{k L-2}+\alpha p_{k L-1}\right) y+$ 
$\left(p_{k L-2}+\alpha p_{k L-1}\right) z>0$. This implies

$$
\left(p_{k L-2}+\alpha p_{k L-1}\right) /\left(q_{k L-2}+\alpha q_{k L-1}\right)=\alpha .
$$

Since the characteristic equation $X(\lambda)$ of $\Phi_{(k ; a, b)}$ is

$$
\begin{aligned}
X(\lambda) & =\lambda^{3}-\left(q_{k L-2}+p_{k L-1}+1\right) \lambda^{2}+\left(q_{k L-2}+p_{k L-1}+1\right) \lambda-1 \\
& =\left[\lambda^{2}-\left(p_{k L-1}+q_{k L-2}\right) \lambda+1\right](\lambda-1) \\
& \equiv P(\lambda)(\lambda-1),
\end{aligned}
$$

and by (2.1), $P\left(q_{k L-2}+\alpha q_{k L-1}\right)=0, X\left(q_{k L-2}+\alpha q_{k L-1}\right)=0$. On the other hand, since $q_{k L-2}+\alpha q_{k L-1}>1$, it is the Perron eigenvalue of $\Phi_{(k ; a, b)}$. Further calculation shows that $(1, \alpha, \alpha)$ is the Perron eigenvector of $\Phi_{(k ; a, b)}{ }^{t r}$. The proof is now complete.

The proof of Theorem 2.1 also proves the following theorem concerning the Bratteli diagram of the dimension group $\left(\boldsymbol{Z}^{2}, P_{\alpha}\right) \bigoplus_{t} \boldsymbol{Z}$ for $\alpha$ a quadratic irrational.

THEOREM 2.3. Let the notation be as above, and let $\alpha$ be a quadratic irrational. The dimension group $\left(\boldsymbol{Z}^{2}, P_{\alpha}\right) \bigoplus_{t} Z$ is isomorphic to the stationary dimension group $\lim \left(\boldsymbol{Z}^{3}, \Phi_{(k ; a, b)}\right)$, (i.e., $\Phi_{(k ; a, b)}$ is a Bratteli diagram of $\left.\left(\boldsymbol{Z}^{2}, P_{\alpha}\right) \bigoplus_{t} \boldsymbol{Z}\right)$, where $a$ and $b$ are any natural numbers, $k$ is an even natural number such that $p_{k L-1}>$ $(a+1), q_{k L-1}>b$.

Suppose we are given a nontotally ordered simple dimension group $G$ with unique state, say $G=\left(\boldsymbol{Z}^{3}, P_{\left(1, \beta_{1}, \beta_{2}\right)}\right)$. Then $G \cong$ $\left(\boldsymbol{Z}^{2}, P_{\alpha}\right) \bigoplus_{t} \boldsymbol{Z}$ for certain $\alpha \in \boldsymbol{R}_{+} \backslash \boldsymbol{Q}$. It is easy to see that $\beta_{1}, \beta_{2} \in \boldsymbol{Q}(\alpha)$, $\alpha \in \boldsymbol{Q}\left(\beta_{1}, \beta_{2}\right)$. Using this observation, we can rewrite Theorem 2.1 as

THEOREM 2.1'. The necessary and sufficient condition for a nontotally ordered simple dimension group $G=\left(\boldsymbol{Z}^{3}, P_{\left(1, \beta_{1}, \beta_{2}\right)}\right)$ to be stationary is that both $\beta_{1}$ and $\beta_{2}$ lie in the same quadratic number. field.

3. Open problems. Motivated by the discussion in $\S 2$, we make the following

Conjecture. There exists a necessary and sufficient condition on the tuple $\alpha_{2}, \cdots, \alpha_{n}$, where $\alpha_{\imath}>0$ for all $i$, and where at least one of them is irrational, for which the automorphism group $\operatorname{AUT}\left(\boldsymbol{Z}^{n}, P_{\left(1, \alpha_{2}, \cdots, \alpha_{n}\right)}\right)$ is nontrivial and contains an element in $\operatorname{GL}(n, \boldsymbol{Z})_{++}$ whose transpose matrix has $\left(1, \alpha_{2}, \cdots, \alpha_{n}\right)$ as its Perron eigenvector. 
(In case when the group is totally ordered, we would believe that the condition is $\alpha_{2}, \cdots, \alpha_{n} \in \boldsymbol{Q}(\lambda)$, where $\lambda>0$ is an integral unit of degree $n$.)

For studying this conjecture, we think the following problem should be considered first:

Problem. Find a necessary and sufficient condition for the totally ordered simple dimension group $\left(\boldsymbol{Z}^{n}, P_{\left(1, \alpha_{2}, \cdots, \alpha_{n}\right)}\right)$ to have a nontrivial automorphism.

While people get stuck for a long time on the search of the periodicity condition for multidimensional continued fraction expansion, to attack this problem for simultaneous Diophantine approximation for a tuple of reals, I think we should, instead of using classical approach, consider the automorphism group of the dimension group with the tuple of reals as the representative of its pure state. Thus we think the answer to the above problems would bring some light to this old periodicity puzzle posed by Lagrange.

\section{REFERENCES}

1. O. Bratteli, Inductive limits of finite dimensional $C^{*}$-algebras, Trans. Amer. Math. Soc., 171 (1972), 195-234.

2. J. Cuntz and W. Krieger, Topological Markov chains with dicyclic dimension groups, preprint, 1979.

3. E. Effros, CBMS Lecture Notes, to appear.

4. E. Effros, D. Handelman and C.-L. Shen, Dimension groups and their affine representations, Amer. J. Math., 102 (1980), 385-407.

5. E. Effros and C.-L. Shen, Approximately finite $C^{*}$-algebras and continued fractions, Indiana Univ. Math. J., 29 (1980), 191-204.

6. E. Effros and C.-L. Shen, Dimension groups and finite difference equations, J. Operator Theory, 2 (1979), 215-231.

7. G. Elliott, On the classification of inductive limits of sequence of semisimple finite dimensional algebras, J. Algebra, 38 (1976), 29-44.

8. - On totally ordered groups and $K_{0}$, Springer Lecture Notes in Mathematics, no. $734,1979$.

9. F. R. Gantmacher, Applications of the Theory of Matrices, Interscience Publishers, 1959.

10. G. H. Hardy and E. M. Wright, An Introduction to the Theory of Numbers, 4th edition, Oxford.

11. M. Pimsner and D. Voiculescu, Imbedding the irrational rotation $C^{*}$-algebra into an $A F$ algebra, preprint, 1979.

12. N. Riedel, Classification of dimension groups and iterating systems, preprint, 1979.

13. C.-L. Shen, A note on the automorphism groups of simple dimension groups. Pacific J. Math., 89 (1980), 199-207.

14. C.-L. Shen, On the classification of the ordered groups associated with approximately finite dimensional C*-algebras, Duke Math. J., 46 (1979), 613-633.

Received March 10, 1980. 



\section{PACIFIC JOURNAL OF MATHEMATICS}

EDITORS

DONALD BABBITT (Managing Editor)

University of California

Los Angeles, CA 90024

Hugo RossI

University of Utah

Salt Lake City, UT 84112

C. C. MOORE and ANDREW OGG

University of California

Berkeley, CA 94720

\section{J. DugundjI}

Department of Mathematics

University of Southern California

Los Angeles, CA 90007

R. FINN and J. MILGRAM

Stanford University

Stanford, CA 94305

\section{ASSOCIATE EDITORS}
R. ARENS
E. F. BECKENBACH
B. H. NEUManN
F. WOLF
K. YoSHIDA

\section{SUPPORTING INSTITUTIONS}

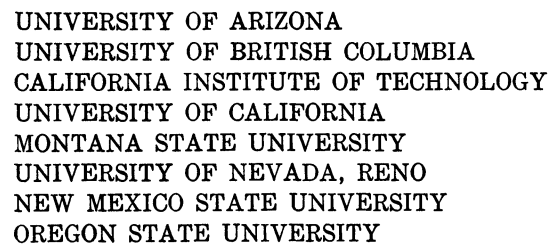

UNIVERSITY OF ARIZONA

UNIVERSITY OF BRITISH COLUMBIA

CALIFORNIA INSTITUTE OF TECHNOLOGY

UNIVERSITY OF CALIFORNIA

MONTANA STATE UNIVERSITY

UNIVERSITY OF NEVADA, RENO

NEW MEXICO STATE UNIVERSITY

OREGON STATE UNIVERSITY

\author{
UNIVERSITY OF OREGON \\ UNIVERSITY OF SOUTHERN CALIFORNIA \\ STANFORD UNIVERSITY \\ UNIVERSITY OF HAWAII \\ UNIVERSITY OF TOKYO \\ UNIVERSITY OF UTAH \\ WASHINGTON STATE UNIVERSITY \\ UNIVERSITY OF WASHINGTON
}

The Supporting Institutions listed above contribute to the cost of publication of this Journal, but they are not owners or publishers and have no responsibility for its content or policies.

Mathematical papers intended for publication in the Pacific Journal of Mathematics should be in typed form or offset-reproduced, (not dittoed), double spaced with large margins. Please do not use built up fractions in the text of the manuscript. However, you may use them in the displayed equations. Underline Greek letters in red, German in green, and script in blue. The first paragraph or two must be capable of being used separately as a synopsis of the entire paper. Please propose a heading for the odd numbered pages of less than 35 characters. Manuscripts, in triplicate, may be sent to any one of the editors. Please classify according to the scheme of Math. Reviews, Index to Vol. 39. Supply name and address of author to whom proofs should be sent. All other communications should be addressed to the managing editor, or Elaine Barth, University of California, Los Angeles, California, 90024.

50 reprints to each author are provided free for each article, only if page charges have been substantially paid. Additional copies may be obtained at cost in multiples of 50 .

The Pacific Journal of Mathematics is issued monthly as of January 1966. Regular subscription rate: $\$ 102.00$ a year (6 Vols., 12 issues). Special rate: $\$ 51.00$ a year to individual members of supporting institutions.

Subscriptions, orders for numbers issued in the last three calendar years, and changes of address shoud be sent to Pacific Journal of Mathematics, P.O. Box 969, Carmel Valley, CA 93924, U.S.A. Old back numbers obtainable from Kraus Periodicals Co., Route 100, Millwood, NY 10546.

\section{PUBLISHED BY PACIFIC JOURNAL OF MATHEMATICS, A NON-PROFIT CORPORATION}

Printed at Kokusai Bunken Insatsusha (International Academic Printing Co., Ltd.). 8-8, 3-chome, Takadanobaba, Shinjuku-ku, Tokyo 160, Japan. 


\section{Pacific Journal of Mathematics}

\section{Vol. 97, No. $2 \quad$ February, 1981}

Patrick Robert Ahern and N. V. Rao, A note on real orthogonal measures . . . . . 249

Kouhei Asano and Katsuyuki Yoshikawa, On polynomial invariants of fibered

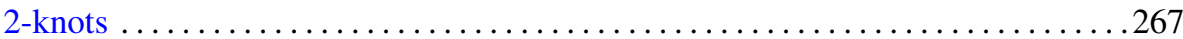

Charles A. Asmuth and Joe Repka, Tensor products for $S L_{2}(\mathscr{K})$. I.

Complementary series and the special representation

Gary Francis Birkenmeier, Baer rings and quasicontinuous rings have a

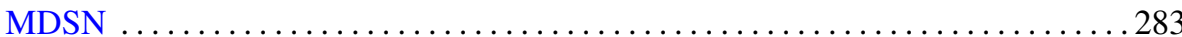

Hans-Heinrich Brungs and Günter Törner, Right chain rings and the generalized

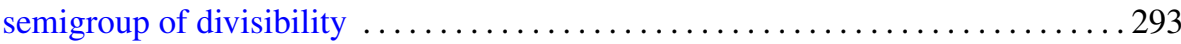

Jia-Arng Chao and Svante Janson, A note on $H^{1} q$-martingales . . . . . . . . . 307

Joseph Eugene Collison, An analogue of Kolmogorov's inequality for a class of

additive arithmetic functions

Frank Rimi DeMeyer, An action of the automorphism group of a commutative ring on its Brauer group

H. P. Dikshit and Anil Kumar, Determination of bounds similar to the Lebesgue

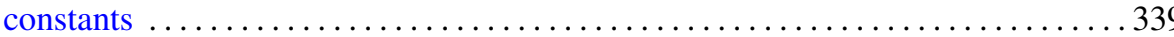

Eric Karel van Douwen, The number of subcontinua of the remainder of the plane

D. W. Dubois, Second note on Artin's solution of Hilbert's 17th problem. Order spaces

Daniel Evans Flath, A comparison of the automorphic representations of GL(3) and its twisted forms

Frederick Michael Goodman, Translation invariant closed $*$ derivations

Richard Grassl, Polynomials in denumerable indeterminates

K. F. Lai, Orders of finite algebraic groups

George Kempf, Torsion divisors on algebraic curves

Arun Kumar and D. P. Sahu, Absolute convergence fields of some triangular matrix methods

Elias Saab, On measurable projections in Banach spaces

Chao-Liang Shen, Automorphisms of dimension groups and the construction of AF algebras

Barry Simon, Pointwise domination of matrices and comparison of $\Phi_{p}$ norms

Chi-Lin Yen, A minimax inequality and its applications to variational inequalities

Stephen D. Cohen, Corrections to: "The Galois group of a polynomial with two indeterminate coefficients"

Phillip Schultz, Correction to: "The typeset and cotypeset of a rank 2 abelian group"

Pavel G. Todorov, Correction to: "New explicit formulas for the $n$th derivative of composite functions"

Douglas S. Bridges, Correction to: "On the isolation of zeroes of an analytic function" 